\section{Profilloses Profil}

_ Kein Künstler präsentiert der Öffentlichkeit ein unfertiges Bild. Erst wenn er es für perfekt hält, enthüllt er es. In der Physiotherapie scheint das anders zu sein: Ende 2010 hat der Berufsverband ZVK zwei Kompetenzprofile veröffentlicht. Ein auserwählter Kreis von fünf Personen hat innerhalb eines Dreivierteljahres zu Papier gebracht, was deutsche Physiotherapeuten aufgrund von sogenannten Ordnungspapieren können. Sie haben ein Profil gezeichnet für einen berufsfachschulisch ausgebildeten und eines für einen akademisch ausgebildeten Physiotherapeuten (๑ S. 11). Kurz nach Veröffentlichung der beiden Kompetenzprofile wandten sich gleich mehrere physiopraxis-Leser an uns. Sie

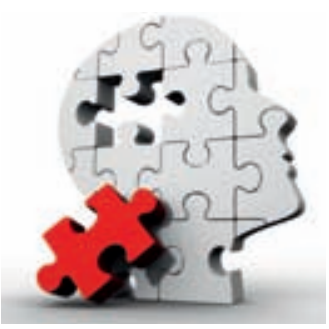
empfinden die Papiere als Degradierung der nicht-

akademischen Physiotherapeuten. Das dadurch erzeugte Bild sei unvollständig, diskussionswürdig und nicht für die Öffentlichkeit geeignet.

- Wir nahmen uns der Sache an, machten uns an die Recherche und kamen zu dem Schluss: Veraltete gesetzliche Rahmenbedingungen aus dem Jahre 1994 sind keine gute Grundlage, um transparent zu machen, was Physiotherapeuten zur optimalen Patientenversorgung heute leisten. Beruft man sich nur auf die Ausbildungs- und Prüfungsverordnung, lässt man den Wandel außer Acht, der in manchen Berufsfachschulen und Bundesländern längst eingesetzt hat. Sie bilden reflektierte Praktiker aus, die wissenschaftliche Erkenntnisse in ihre Therapie einfließen lassen.

_ Uns liegt die Qualität und Weiterentwicklung der Physiotherapieausbildung sehr am Herzen. Umso mehr sind wir irritiert darüber, dass der ZVK im Profil für den Physiotherapteuten nur den Mindeststandard beschreibt. Zur Orientierung dient es wenig. Es wird Politik und Ärzteschaft nicht davon überzeugen, Physiotherapeuten mehr Verantwortung zu übertragen. Und es ist ein Freibrief für schlechte Ausbilder. Wir vermissen das Kämpferische und Zukunftsorientierte, das den Berufsstand voranbringt. Förderlich ist ein Bild, hinter dem Therapeuten stehen, und das sie gerne in der Öffentlichkeit zeigen: Seht her, das kann ich!

Herzlich, Ihre

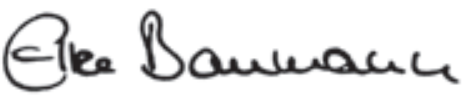

Physiotherapeuten haben Profil, auch wenn das in den Kompetenzpapieren des ZVK nicht zur Geltung kommt.

\section{ZU GEWININEN}

Für jede physiopraxis organisiert unsere gute Fee attraktive Gewinne für Sie. Möchten Sie den einen oder anderen ergattern, klicken Sie ins Internet unter: www.thieme.de/ physioonline > "physioexklusiv“. Und das gibt es in dieser Ausgabe zu gewinnen:

Kursplatz

Kursreihe „FlexotapingMyofasziales Taping“"

Seite 32

Kursplatz „Lymphtaping“ Seite 64

Bücher

2-mal „Heterogenität im

Klassenzimmer"

Seite 39

4-mal „Untersuchen

in der Physiotherapie“

Seite 48

2-mal „Indien -

Küche und Kultur"

Seite 52

und außerdem

20 Rollen Flexotape und eine Tapeschere

Seite 32

2-mal drei Antistressbälle 